\title{
Intervalo QT Corrigido e Fatores de Risco Tradicionais para Doença Aterosclerótica em Pacientes com Diabetes Tipo 1
}

\begin{abstract}
RESUMO
Visando avaliar o intervalo QTc e sua relação com variáveis clínicas, laboratoriais e com suscetibilidade da LDL à oxidação in vitro em pacientes com DM1, estudamos 40 diabéticos e 33 não diabéticos com idades de 24,83 \pm 10,21 e 23,51 $\pm 7,28$ anos, respectivamente, pareados por sexo, idade e índice de massa corporal (IMC). Avaliamos controle metabólico, apolipoproteínas A e B, coeficiente de oxidação da LDL por espectrofotometria e eletrocardiograma (ECG). O intervalo QTc foi calculado pela fórmula de Bazett. Não houve diferença no QTc entre os grupos dos DM1 e dos não diabéticos $(394,43 \pm 19,98 \mathrm{~ms}$ vs. $401,31 \pm 17,83 \mathrm{~ms} ; \mathrm{p}=0,2065)$. Cinco diabéticos apresentavam QTc aumentado $(396,76 \pm 14,63 \mathrm{~ms}$ vs. $429,75 \pm 1,89 \mathrm{~ms} ; \mathrm{p}<0,001)$ e menores níveis de apolipoproteína A que os demais diabéticos $(74,60 \pm 25,42 \mathrm{mg} / \mathrm{dL}$ vs. $113,64 \pm$ $29,79 \mathrm{mg} / \mathrm{dL} ; \mathrm{p}=0,011$ ). Na amostra total, houve correlação entre OTc e IMC (rho $=-0,288 ; p=0,045)$, glicemia pós-prandial $(r h o=0,357 ; p=0,016)$ e coeficiente de oxidação 3 h (Cox3h) ( $r=-0,293 ; p=0,039)$. Nos diabéticos, encontramos correlação entre QTc e triglicerídeos ( $r h o=-0,420 ; p=0,023)$ e Cox3h $(r=-0,427$; $p=0,021)$. Embora não tenhamos encontrado diferença entre o OTc dos diabéticos e não diabéticos estudados, houve correlação com marcadores de risco para a doença aterosclerótica. Entretanto, ainda são necessários mais estudos para estabelecer o real valor preditivo do QTc para esta doença nos pacientes com DM1. (Arq Bras Endocrinol Metab 2007;51/7:1153-1159)
\end{abstract}

Descritores: Diabetes tipo 1; Intervalo QT corrigido; LDL oxidada

\section{ABSTRACT}

QTc Interval and Traditional Risk Factors to Atherosclerotic Disease in Patients With Type 1 Diabetes.

To evaluate the QTc interval and its relation with clinical, laboratorial variables and LDL susceptibility to in vitro oxidation in patients with type $1 \mathrm{DM}$, we studied 40 diabetics and 33 non diabetics with $24.83 \pm 10.21$ and $23.51 \pm 7.28$ years old, respectively matched by sex, age and body mass index (BMI). We evaluated metabolic control, A and B apolipoproteins, LDL oxidation coefficient for spectrophotometry and electrocardiogram (ECG). Interval QTc was calculated by the Bazett's formula. There was no difference in QTc between diabetic and non diabetic groups $(394.43 \pm 19.98 \mathrm{~ms}$ versus $401.31 \pm 17.83 \mathrm{~ms} ; \mathrm{p}=0.2065)$. Five diabetics showed increased QTc $(396.76 \pm 14.63 \mathrm{~ms}$ versus $429.75 \pm 1.89 \mathrm{~ms} ; \mathrm{p}<0.001)$ and lesser $A$ apolipoprotein levels than rest of diabetic group $(74.60 \pm 25.42 \mathrm{mg} / \mathrm{dL}$ versus $113.64 \pm 29.79 \mathrm{mg} / \mathrm{dL} ; \mathrm{p}=0,011)$. In pooled sample, there was correlation between QTc and BMI ( $r$ ho $=-0.288 ; p=0.045$ ), pot-prandial glycemia ( $r$ o $=0.357$; $p=0.016)$ and $3 \mathrm{~h}$ oxidation coefficient $(\mathrm{OxC} 3 \mathrm{~h})(\mathrm{r}=-0.293 ; \mathrm{p}=0.039)$. In diabetics, there was correlation between QTc and triglycerides ( $r$ o $=-0.420 ; p=0.023$ ) and $\mathrm{OxC} 3 \mathrm{~h}(\mathrm{r}=-0.427 ; \mathrm{p}=0.021)$. Although there was no difference between QTc of diabetics and the non diabetics subjects studied, there was correlation with risk factors for the atherosclerotic disease. Further studies are necessary to establish the real predictive value of QTc for this type of disease in the patients with type 1 DM. (Arq Bras Endocrinol Metab 2007;51/7:1153-1159)

Keywords: Type 1 diabetes; Corrected OT interval; Oxidized LDL

\section{artigo original}

\author{
Simone HenRiques de Castro \\ hugo C. Castro Faria-Neto \\ MARILIA DE BRITO GOMES
}

Serviço de Diabetes do Hospital Universitário Pedro Ernesto (SHC \& MBG) e Laboratório de Imunofarmacologia da FIOCRUZRJ (HCCFN), Rio de Janeiro, RJ.
Recebido em 23/08/05 Revisado em 07/08/06 Aceito em 04/12/06 
A S DOENCCAS ASSOCIADAS COM A aterosclerose são consideradas atualmente patologias inflamatórias crônicas caracterizadas pelo acúmulo excessivo de macrófagos derivados de monócitos na parede arterial (1), e são a principal causa de mortalidade na população mundial. No Brasil, os últimos dados estatísticos são de 1995 e apontam o infarto agudo do miocárdio e a doença cerebrovascular como responsáveis por $6,05 \%$ e $9,14 \%$, respectivamente (2). Essas mesmas doenças foram a causa de 7,0\% das mortes, cada uma, nos Estados Unidos da América no ano de 1999 (2). Nos pacientes com diabetes, a doença cardiovascular também é a principal causa de mortalidade, perfazendo $44 \%$ do total de casos de morte nos pacientes com diabetes tipo 1 (DM 1), seguida pela doença renal $(21 \%)(3)$.

$\mathrm{O}$ estresse oxidativo, representado pelo o aumento da formação de radicais livres, destaca-se como fator inicial de várias reações que culminam com o desencadeamento do processo aterosclerótico. Diversas situações podem aumentar a formação de radicais livres, como a hiperlipidemia, o diabetes, o tabagismo e a hipertensão (4). No caso dos pacientes com DMl, esse processo tem início ainda na infância, segundo Järvisalo e cols. (5), que demonstraram que mesmo as crianças já apresentam espessamento do complexo íntima-média das carótidas. O LDL oxidado tem sido caracterizado como um fator crítico no processo aterosclerótico tanto em modelos animais como em humanos (6). A modificação oxidativa da LDL confere propriedades específicas pró-aterogênicas a esta molécula, a qual tem ação na célula endotelial, nos macrófagos e células musculares lisas.

Algumas anormalidades detectadas no eletrocardiograma (ECG) representativas de alterações na repolarização ventricular, como o intervalo QT prolongado, são marcadores bem estabelecidos do risco de mortalidade na população geral $(7,8)$. Festa e cols. (9) demonstraram que em indivíduos não diabéticos sem doença arterial coronariana (DAC) há significativa re- lação entre a duração do intervalo QT com a aterosclerose carotídea. Em função da forte correlação da DAC e da aterosclerose carotídea, já avaliada histologicamente e clinicamente, o intervalo QT pode ser um marcador não somente para a doença aterosclerótica carotídea, mas também para a aterosclerose coronariana $(10,11)$. O valor da análise das alterações de repolarização ventricular no ECG como método não invasivo de estratificação de risco já foi estabelecido também para pacientes com diabetes tipo 2 (12), entretanto em pacientes com DM 1 os dados são controversos (13-15).

O presente trabalho tem como objetivo avaliar o intervalo QTc e sua relação com variáveis clínicodemográficas, e com o controle metabólico, os níveis de apolipoproteínas A e B e a suscetibilidade da lipoproteína de baixa densidade (LDL) à oxidação in vitro em pacientes com DMl acompanhados regularmente no ambulatório de Diabetes do Hospital Universitário Pedro Ernesto.

\section{PACIENTES E MÉTODOS}

Foram recrutados 40 pacientes com DMl acompanhados regularmente no ambulatório de Diabetes do Hospital Universitário Pedro Ernesto (HUPE) - UERJ e 33 não diabéticos, pareados por sexo, idade e índice de massa corporal (IMC), após assinatura do termo de consentimento previamente aprovado pelo comitê de ética do HUPE. As características das duas amostras no momento da coleta dos exames estão na tabela 1 .

Os critérios de exclusão para os diabéticos foram tabagismo, etilismo, presença de nefropatia diabética, infecção sistêmica e/ou o uso de medicamentos que pudessem alterar os resultados da determinação da suscetibilidade do LDL à oxidação, como os inibidores da ECA, sulfato ferroso e complexos vitamínicos e de sais minerais contendo vitaminas $\mathrm{C}$ e $\mathrm{E}$ e $\mathrm{Zn}, \mathrm{Se}$, Fe e $\mathrm{Cu}$, e presença de retinopatia e nefropatia diabéticas e neuropatia diabética clínica. No grupo dos não diabéticos, esses critérios incluíam também a presença de familiares diretos portadores de diabetes mellitus.

Tabela 1. Características do grupo de não diabéticos e de DM1 no momento da coleta dos exames.

\begin{tabular}{lccc}
\hline Variáveis & DM 1 & Não diabéticos & p \\
$\mathrm{N}$ & 40 & 33 & - \\
Sexo (F/M) & $17 / 23$ & $18 / 15$ & - \\
Idade (anos) & $24,83 \pm 10,21$ & $23,51 \pm 7,28$ & \\
Idade no diagnóstico (anos) & $16,18 \pm 8.99$ & - & - \\
Duração da doença (anos) & $8,68 \pm 5,91$ & - & - \\
Índice de massa corporal $\left(\mathrm{kg} / \mathrm{m}^{2}\right)$ & $22,29 \pm 3,14$ & $23,36 \pm 3,79$ & \\
PAS (mmHg) & $110,00 \pm 10,64$ & $110,12 \pm 11,46$ & 0,8682 \\
PAD (mmHg) & $69,22 \pm 9,68$ & $67,94 \pm 6,84$ & 0,5486 \\
\hline PAS = pressão arterial sistólica, PAD $=$ pressão arterial diastólica &
\end{tabular}


Os participantes foram submetidos à coleta de sangue após 12 horas de jejum e 2 horas após café da manhã habitual. Os exames realizados foram glicemia de jejum (GJ) e pósprandial (GPP) (glicose oxidase), colesterol total (CT), colesterol HDL, triglicerídeos (TG) e ácido úrico (AU) através de reações colorimétricas com leitura pelo aparelho CobasMira (Roche), hemoglobina glicada (HbAlc - HPLC com leitura pelo aparelho Merck Hitachi 9100 - VR 2,6 a 6,2\% e coeficientes de variação intra e interensaio menor que $1 \%$ ), apolipoproteínas Al e B por imunoturbidimetria (Behring Turbidimeter, Alemanha), sendo os valores de referência 115 a $220 \mathrm{mg} / \mathrm{dl}$ (sensibilidade de $30 \mathrm{mg} / \mathrm{dl}$ ) e 115 a $220 \mathrm{mg} / \mathrm{dl}$ (sensibilidade de $30 \mathrm{mg} / \mathrm{dl}$ ) e os coeficientes intra e interensaio $5,0 \%$ e $4,8 \%$ e $4,2 \%$ e $4,0 \%$, respectivamente. O LDL foi calculado pela fórmula de Friedwald (16).

Os pacientes tiveram seu controle glicêmico classificado de acordo com os critérios descritos por Chase (divisão do valor da HbAlc do paciente pelo valor do limite superior da normalidade do método) da seguinte forma: bom controle (índice $<1,33$ ), controle regular (índice $\geq$ 1,33 e $<1,5$ ) e péssimo controle (índice $\geq 1,55$ ) (17).

Todos os pacientes coletaram 3 amostras de urina noturna em um período de 3 meses. Eles foram orientados a desprezar a urina das 20 horas e coletar toda a urina até 6 horas do dia seguinte para a determinação da taxa de excreção de albumina (EUA). O volume urinário foi aliquotado e estocado em frascos de vidro a $70^{\circ} \mathrm{C}$ até a análise. A concentração urinária de albumina foi determinada por radioimunoensaio (Diagnostic, Califórnia, Estados Unidos, sensibilidade de $0,3 \mu \mathrm{g} / \mathrm{mL}$ ) com coeficientes de variação intra e interensaio de $8,7 \%$ e $8,3 \%$, respectivamente. Baseado na EUA, somente pacientes com normoalbuminúria (EUA < $20 \mu \mathrm{g} / \mathrm{min}$ em duas das três amostras de urina) (18) foram incluídos. Os pacientes com DMl foram submetidos à fundoscopia com dilatação das pupilas através de oftalmoscopia pelo mesmo oftalmologista.

Para a análise da suscetibilidade do LDL à oxidação in vitro foram coletados $20 \mathrm{~mL}$ de sangue a vácuo em tubos com EDTA, os quais foram centrifugados a $4{ }^{\circ} \mathrm{C}$ por 20 minutos a $800 \mathrm{x}$ g. Após a separação do plasma, o mesmo foi imediatamente processado para o isolamento do LDL seguindo as etapas descritas: ajuste da densidade para 1,3 $\mathrm{g} / \mathrm{mL}$ pela adição de brometo de potássio $(4,5 \mathrm{~g}$ de $\mathrm{KBr}$ a cada $9 \mathrm{~mL}$ de plasma) com posterior preparo dos tubos de ultracentrifugação com $20 \mathrm{~mL}$ de solução salina a $0,9 \%$ e os $9 \mathrm{~mL}$ de plasma anteriormente preparados. Esses tubos foram então centrifugados a $4^{\circ} \mathrm{C}$ por 3 horas a $150.00 \mathrm{x} \mathrm{g}$. Após a ultracentrifugação, a banda de lipoproteínas com densidade entre 1,019 e $1,063 \mathrm{~g} / \mathrm{mL}$, compatível com a do LDL, foi coletada $(19,20)$. Esse material teve sua concentração protéica dosada pelo método do Biureto (21) e ajustada para $0,2 \mathrm{mg} / \mathrm{mL}$. Ao material obtido foi adicionado sulfato de cobre $\left(\mathrm{CuSO}_{4}\right) 20 \mathrm{mM}$ na proporção de $1 \mu \mathrm{L}$ para cada $1 \mathrm{~mL}$ de LDL e esta solução foi colocada em banho a $37^{\circ} \mathrm{C}$ por 24 horas.

A avaliação do LDL oxidado foi feita de forma indireta através do cálculo do coeficiente de oxidação desta partícula $(22,23)$, o qual utiliza em sua fórmula a absorvância em 3 comprimentos de onda da luz UV, a saber: $205 \mathrm{~nm}, 232 \mathrm{~nm}$ e $280 \mathrm{~nm}$.

Foram realizadas leituras no espectrofotômetro Schimadzu nos comprimentos de onda acima mencionados nos momentos pré-adição e 1, 3, 6 e 24 h após a adição do $\mathrm{CuSO}_{4}$, para avaliar a suscetibilidade do LDL ao processo oxidativo. O coeficiente de oxidação foi calculado através da seguinte fórmula: Abs 205 - Abs 280 / Abs 232 - Abs 280, onde Abs 205 = leitura das duplas ligações dos ácidos graxos poliinsaturados dos fosfolipídeos do LDL; Abs 232 = leitura dos dieno conjugados; e Abs 280 = leitura da fração protéica do LDL.

Foi realizado eletrocardiograma (ECG) de 12 derivações em repouso, o qual foi classificado segundo o protocolo de Minesota (24) por dois observadores em esquema duplo-cego. A partir dessa codificação, os pacientes foram definidos nas seguintes categorias: sem doença coronariana, doença coronariana possível ou definida ou infarto do miocárdio possível ou definido (25).

O intervalo QT corrigido foi calculado através da fórmula de Bazett (15), sendo considerado o valor normal máximo de $440 \mathrm{~ms}$ para as mulheres e de $424 \mathrm{~ms}$ para os homens (26).

\section{ANÁLISE ESTATÍSTICA}

Os resultados de todas as variáveis analisadas foram apresentados como média e desvio-padrão. Foi utilizado o teste $t$ Student não pareado para comparação das médias entre os dois grupos analisados. As variáveis de distribuição não Gaussiana foram logaritmizadas antes da comparação entre os grupos. Para testar as correlações entre o intervalo QT corrigido e as variáveis de controle metabólico e a suscetibilidade do LDL à oxidação in vitro, utilizamos a correlação de Pearson e de Spearman de acordo com a distribuição das variáveis.

\section{RESULTADOS}

Os resultados do controle metabólico, bem como das apolipoproteínas A e B, estão na tabela 2. Observamos que o controle glicêmico dos pacientes estudados era regular e que não houve diferença estatisticamente significativa no perfil lipídico dos dois grupos avaliados. Com relação às apolipoproteínas, os pacientes com $\mathrm{DMl}$ apresentaram níveis mais elevados de apo Al que os não diabéticos $(110,74 \pm 31,63 \mathrm{mg} / \mathrm{dL}$ vs. 94,06 $\pm 24,47$ $\mathrm{mg} / \mathrm{dL} ; \mathrm{p}=0,0188)$. Os níveis de ácido úrico foram menores nos diabéticos que nos não diabéticos $(3,62 \pm$ $1,03 \mathrm{mg} / \mathrm{dL}$ vs. $4,75 \pm 1,34 \mathrm{mg} / \mathrm{dL} ; \mathrm{p}=0,0001)$. 
Tabela 2. Variáveis de controle metabólico e apolipoproteínas A e B.

\begin{tabular}{lccc}
\hline Variáveis & DM 1 & Não diabéticos & P \\
GJ (mg/dL) & $167,13 \pm 107,31$ & $79,16 \pm 8,25$ & - \\
GPP (mg/dL) & $216,97 \pm 117,52$ & $96,56 \pm 17,11$ & - \\
HbA1c $(\%)$ & $8,77 \pm 2,28$ & $4,56 \pm 0,63$ & - \\
IC $(\%)$ & $1,41 \pm 0,36$ & $0,73 \pm 0,09$ & - \\
CT $(\mathrm{mg} / \mathrm{dL})$ & $165,68 \pm 32,31$ & $180,70 \pm 43,39$ & 0,1049 \\
HDL $(\mathrm{mg} / \mathrm{dL})$ & $46,05 \pm 11,07$ & $45,70 \pm 11,49$ & 0,9031 \\
LDL $(\mathrm{mg} / \mathrm{dL})$ & $104,25 \pm 25,13$ & $116,09 \pm 37,83$ & 0,1301 \\
TG $(\mathrm{mg} / \mathrm{dL})$ & $82,23 \pm 39,05$ & $94,24 \pm 54,12$ & 0,3137 \\
$\mathrm{CT} / \mathrm{HDL}$ & $3,75 \pm 0,76$ & $4,14 \pm 1,35$ & 0,1531 \\
$\mathrm{LDL} / \mathrm{HDL}$ & $2,36 \pm 0,67$ & $2,68 \pm 1,11$ & 0,3956 \\
Apo A1 (mg/dL) & $110,74 \pm 31,63$ & $94,06 \pm 24,47$ & 0,0188 \\
Apo B (mg/dL) & $88,39 \pm 29,84$ & $94,42 \pm 36,02$ & 0,9291 \\
AU (mg/dL) & $3,62 \pm 1,03$ & $4,75 \pm 1,34$ & 0,0001 \\
\hline GJ = glicemia de jejum, GPP = glicemia pós-prandial, IC = índice de controle, CT \\
$=$ colesterol total, HDL = lipoproteína de alta densidade, LDL = lipoproteína de \\
baixa densidade, TG = triglicerídeos, apo A1 = apolipoproteína A1, apo B = \\
apolipoproteína B, AU = ácido úrico
\end{tabular}

Os pacientes com DMl apresentaram média de coeficiente de oxidação basal semelhante à dos não diabéticos. Entretanto, após a adição de sulfato de cobre, houve oxidação mais precoce nos pacientes diabéticos que nos não diabéticos (tabela 3 , figura 1 ).

De acordo com o Código de Minesota, nenhum paciente apresentava doença coronariana possível ou definida ou infarto do miocárdio possível ou definido. A análise do intervalo QTc mostrou não haver diferença entre os grupos dos DMl e dos não diabéticos $(394,43 \pm 19,98 \mathrm{~ms}$ vs. 401,31 $\pm 17,83 \mathrm{~ms}$; $\mathrm{p}=0,2065)$. No grupo dos não diabéticos, todos apresentaram o intervalo QTc dentro da normalidade, entretanto 5 diabéticos estavam com esse intervalo acima do limite da normalidade $(396,76 \pm 14,63 \mathrm{~ms}$ vs. $429,75 \pm 1,89 \mathrm{~ms} ; \mathrm{p}<0,001)$.

Os cinco diabéticos tipo 1 que apresentavam o intervalo QTc acima do limite da normalidade possuíam diferenças em relação aos demais diabéticos estudados no que diz respeito aos níveis de apolipoproteína A $(74,60 \pm 25,42 \mathrm{mg} / \mathrm{dL}$ vs. $113,64 \pm$ $29,79 \mathrm{mg} / \mathrm{dL} ; \mathrm{p}=0,011)$. Não houve correlação entre a concentração de apo A e o QTc nestes pacientes ( rho $=-0,632 ; \mathrm{p}=0,368)$.

Ao avaliarmos a correlação entre o QTc e as demais variáveis na amostra total, observamos dados significativos com IMC ( $\mathrm{rho}=-0,288 ; \mathrm{p}=0,045)$, GPP (rho $=0,357 ; \mathrm{p}=0,016)$ e Cox $3 \mathrm{~h}(\mathrm{r}=-0,293 ; \mathrm{p}$ $=0,039)$. Ao analisarmos apenas o grupo dos diabéticos, encontramos correlação significativa somente entre QTc e TG (rho = -0,420; p = 0,023) e Cox3h $(r=-0,427 ; p=0,021)$.

\section{DISCUSSÃo}

As doenças cardiovasculares são a principal causa de mortalidade em pacientes com diabetes mellitus (3). Vários estudos têm sido realizados visando avaliar o valor preditivo de métodos diagnósticos não invasivos para essas patologias $(8,10-15)$. A validade do intervalo QTc como preditor de doença cardiovascular já foi bem estabelecida em relação à população não diabética $(10,11,27)$ e aos diabéticos tipo 2 (12). Entretanto, os dados na literatura ainda são controversos no que diz respeito ao seu valor prognóstico em pacientes com diabetes tipo 1 (13-15).

O nosso estudo teve como objetivo a avaliação do intervalo QTc em uma amostra composta por indivíduos não diabéticos e diabéticos tipo 1 e a investigação de sua correlação com variáveis de controle metabólico, apolipoproteínas A e B e a suscetibilidade da LDL à oxidação in vitro, fatores relacionados com o maior risco de desenvolvimento de doenças ateroscleróticas. Não analisamos o valor preditivo do QTc como fator prognóstico de mortalidade por doença cardiovascular, já que nosso estudo foi de corte transverso e os pacientes não apresentavam alterações eletrocardiográficas sugestivas de tais enfermidades segundo o Código de Minesota (24), nem foram submetidos a métodos mais invasivos de investigação dessas doenças. Nossos resultados mostraram não haver diferença significativa entre esse intervalo nos grupos dos pacientes com DMl e dos não diabéticos. Entretanto, encontramos 5 pacientes com DMl com intervalo QTc prolongado. Apesar de 


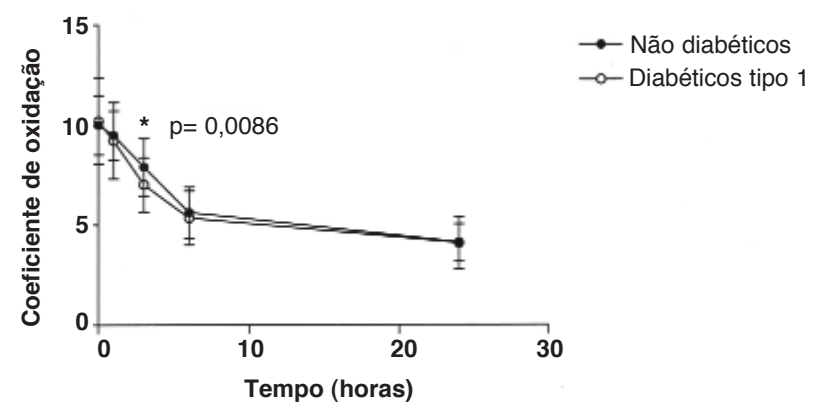

Figura 1. Curva do coeficiente de oxidação (COx) do LDL mostrando diferença significativa entre diabéticos tipo 1 e não diabéticos 3 horas após adição de sulfato de cobre

nossa amostra de pacientes com DMl apresentar níveis maiores de apolipoproteína A que os não diabéticos, os quatro indivíduos com o intervalo QTc prolongado diferiam dos demais diabéticos por possuírem menores concentrações dessa apolipoproteína. Como Snehalatha e cols. evidenciaram, as apolipoproteínas A e B são mais comumente e fortemente relacionadas com a presença de doença arterial coronariana comprovada angiograficamente que os níveis de LDL em uma população com DM2 (28). Caso isso seja verdadeiro também em relação aos pacientes com DMl, a presença de prolongamento de QTc em pacientes com menores níveis de apo A justificaria a maior prevalência de doença aterosclerótica nos diabéticos tipo 1 que nos não diabéticos, mesmo sem os primeiros apresentarem os fatores de risco clássicos para essas doenças, como aumento do LDL ou dos TG ou diminuição de $\operatorname{HDL}(5,30)$.

Além da concentração dos níveis das apolipoproteínas A e B, os estudos mostraram haver alteração também da estrutura das mesmas nos pacientes com diabetes (31). Essas alterações associadas a outras extrínsecas à molécula de LDL, por exemplo a diminuição de substâncias antioxidantes como o ácido úrico nos diabéticos (32) e a formação aumentada de radicais livres, estão envolvidos com a maior suscetibilidade da LDL à oxidação $(33,34)$. O LDL oxidado tem sido caracterizado como um fator crítico no processo aterosclerótico, tanto em modelos animais como em humanos (6). A modificação oxidativa do LDL confere propriedades específicas pró-aterogênicas a essa molécula, a qual tem ação na célula endotelial, nos macrófagos e células musculares lisas.

Os dados na literatura são controversos no que diz respeito ao comportamento do LDL de pacientes com DMl frente ao stress oxidativo (34-36). Em nossa amostra observamos uma oxidação mais precoce da LDL dos pacientes com DMl, quando exposta ao
Tabela 3. Coeficientes de oxidação (Cox) do LDL.

\begin{tabular}{lccc}
\hline Cox & DM 1 & Não diabéticos & P \\
Basal & $10,20 \pm 2,17$ & $10,00 \pm 1,48$ & 0,6485 \\
Cox 1h & $9,23 \pm 1,92$ & $9,49 \pm 1,23$ & 0,1480 \\
Cox 3h & $7,02 \pm 1,35$ & $7,90 \pm 1,43$ & 0,0086 \\
Cox 6h & $5,34 \pm 1,34$ & $5,62 \pm 1,28$ & 0,3705 \\
Cox 24h & $4,08 \pm 1,33$ & $4,11 \pm 0,91$ & 0,4152
\end{tabular}

Basal = coeficiente de oxidação basal (pré-adição de sulfato de cobre); Cox $1 \mathrm{~h}=$ coeficiente de oxidação 1 hora após adição de sulfato de cobre; Cox $3 \mathrm{~h}=$ coeficiente de oxidação 3 hora após adição de sulfato de cobre; Cox $6 \mathrm{~h}=$ coeficiente de oxidação 6 hora após adição de sulfato de cobre; Cox $24 \mathrm{~h}$ = coeficiente de oxidação 24 hora após adição de sulfato de cobre.

agente oxidante, que a dos não diabéticos, o que está de acordo com os dados de Tsai e cols. (35).

Ao analisarmos a correlação do intervalo QTc com as variáveis clínico-demográficas, de controle metabólico, as apolipoproteínas A e B e o coeficiente de oxidação na população total do estudo, observamos relação direta com a GPP e relação inversa com o IMC e o Cox3h. Essas análises apenas no grupo dos diabéticos revelaram relação inversa com o TG e o Cox3h.

A relação direta entre a GPP e o intervalo QTc é um dado que pode associar essa medida com a doença cardiovascular, já que hoje em dia há evidências de que a GPP seja um fator de risco independente para essa doença em diabéticos tipo 2 (38-40). Em pacientes com diabetes tipo 1 , não há estudos avaliando a importância da GPP nas complicações microvasculares e macrovasculares. Entretanto, a importância da hiperglicemia como fator determinante de doenças microvasculares já foi mostrada pelo Diabetes Complications Control Trial (DCCT) (41). E, de acordo com diferentes estudos, há associação entre a hiperglicemia e o espessamento do complexo íntima-média em pacientes com DMl (42) e DM2 (43). Sugere-se que o espessamento do complexo íntima-média seja um marcador sensível para doença coronariana e cerebrovascular e, segundo Järvisalo e cols., mesmo as crianças com DMl podem apresentar esta alteração em suas paredes arteriais (5).

A relação inversa com o coeficiente de oxidação 3 horas após a adição de sulfato de cobre mostra que quanto menor for este coeficiente - portanto mais oxidado o LDL - maior será o intervalo QTc. Essa correlação com um fator crítico no processo aterosclerótico, como o LDL oxidado, ainda não havia sido avaliada na literatura.

A correlação inversa com o IMC é um dado que pode ser um viés estatístico na análise dos dados em função de nossos pacientes com diabetes tipo 1 terem 
IMC menor que os não diabéticos, já que a obesidade está relacionada com maior risco de doença cardiovascular (44).

A relação inversa entre os níveis de triglicerídeos e o intervalo QTc nos pacientes com DMl também pode representar um viés estatístico, visto que a hipertrigliceridemia é um fator de risco estabelecido para doença aterosclerótica por estar relacionada com diminuição dos níveis de HDL, aumento da concentração de lipoproteínas remanescentes e LDL pequeno e denso e aumento da trombogênese (45).

Concluímos que, em nossa população, não houve diferença entre o intervalo QTc de pacientes com DMl sem complicações microvasculares e sem doença aterosclerótica clínica e o de não diabéticos, apesar de 5 pacientes com DMl terem apresentado essa medida acima do limite da normalidade. Houve correlação direta com a glicemia pós-prandial na população total e com o Cox3h tanto na população geral quanto na análise isolada dos pacientes com DMl. Apesar de não termos nos proposto a avaliar o valor preditivo do QTc, essas correlações com fatores de risco para a doença aterosclerótica sugerem que o QTc talvez possa ser um marcador indireto de risco para a doença aterosclerótica subclínica. São necessários, entretanto, estudos prospectivos a fim de estabelecer o real valor preditivo do QTc nos pacientes com DMl.

\section{REFERÊNCIAS}

1. Ross R. Atherosclerosis - an inflammatory disease. N Engl J Med 1999;340:115-26.

2. World Health Report. Available at: <http://www.who.int/whr>. Accessed in August 2004.

3. Morrish NJ, Wang S-L, Stevens LK, Fuller JH, Keen H. The WHO Multinational Study Group: Mortality and causes of death in the WHO multinational study of vascular disease in diabetes. Diabetologia 2001:44(suppl. 2):S14-21.

4. Kunsch C, Medford RM. Oxidative stress as a regulator of gene expression in the vasculature. Circ Res 1999;85:75366.

5. Järvisalo MJ, Putto-Laurila A, Jartti L , Lehtimäki T, Solakivi $T$, Rönnemaa T, et al. Carotid artery intima-media thickness in children with type 1 diabetes. Diabetes 2002;51:493-8.

6. Chisolm GM, Steinberg D. The oxidative modification hypothesis of atherogenesis: an overview. Free Radical Biol Med 2000;28:1815-26.

7. Daviglus ML, Liao Y, Greenland P, Dyer AR, Liu K, Xie X, et al. Association of nonspecific minor ST-T abnormalities with cardiovascular mortality: the Chicago Western Electric Study. JAMA 1999;281:530-6.

8. Okin PM, Devereux RB, Howard BV, Fabsitz RR, Lee ET, Welty TK. Assessment of QT interval and QT dispersion for prediction of all-cause and cardiovascular mortality in American Indians: the Strong Heart Study. Circulation 2000;101: 616.

9. Festa A, D'Agostino R Jr, Rautaharju P, O'Leary DH, Rewers M, Mykkanen $L$, et al. Is QT interval a marker of subclinical atherosclerosis in nondiabetic subjects? The insulin resistance atherosclerosis study (IRAS). Stroke 1999;30:1566-71.
10. Burke GL, Evans GW, Riley WA, Sharrett AR, Howard G, Barnes RW, et al. Arterial thickness is associated with prevalent cardiovascular disease in middle-aged adults: the Atherosclerosis Risk in Communities (ARIC) Study. Stroke 1995;26:386-91.

11. Mitchell JR, Schwartz CJ. Relationship between arterial disease at different sites. BMJ 1962;1:1293-301.

12. Okin PM, Devereux RB, Lee ET, Galloway JM, Howard BV. Electrocardiographic repolarization complexity and abnormality predict all-cause and cardiovascular mortality in diabetes. The Strong Heart Study. Diabetes 2004;53:434-40.

13. Olson JC, Erbey JR, Williams KV, Becker DJ, Edmundowicz D, Kelsey SF, et al. Subclinical atherosclerosis and estimated glucose disposal rate as predictors of mortality in type 1 diabetes. Ann Epidemiol 2002;12:331-7.

14. Rathman W, Ziegler D, Jahnke M, Haastert B, Gries FA. Mortality in diabetic patients with cardiovascular autonomic neuropathy. Diabetic Med 1993;10:820-4.

15. Sawicki PT, Bender R, Berger M. Prolonged QT interval as a predictor of mortality in diabetic nephropathy. Diabetologia 1996;39:77-81.

16. Friedwald WT, Levy R, Fredrickson DS. Estimations of serum low-density lipoprotein cholesterol without use of preparative ultracentrifuge. Clin Chem 1972;18:499-502.

17. Chase HP, Jackson WE, Hoops SL, Cockerham RS, Archer PG, O'Brien D. Glucose control and the renal and retinal complications of insulin-dependent diabetes. JAMA 1989;261:1155-60.

18. Mogensen CE, Keane WF, Bennett PH, Jerums G, Parving HH, Passa $P$, et al. Prevention of diabetic renal disease with special reference to microalbuminuria. Lancet 1995;346:1080-4.

19. Chung BH, Wilkinson T, Geer JC, Segrest JP. Preparative and quantitative isolation of plasma lipoproteins: rapid, single discontinuous density gradient ultracentrifugation in a vertical rotor. J Lipid Res 1980;21:284-91.

20. Heery JM, Kozak M, Stafforini DM, Jones DA, Zimmerman GA, Mclntyre TM. Oxidatively modified LDL contains phospholipids with platelet-activating factor-like activity and stimulates the growth of smooth muscle cells. J Clin Invest 1995; $96: 2322-30$

21. Gornall AG, Bardawill CJ, David MM. Determination of serum proteins by means of the Biuret reaction. $\mathbf{J}$ Biol Chem 1949; 177:751-66

22. Kim BS, LaBella FS. Comparison of analytical methods for monitoring autoxidation profiles of authentic lipids. J Lipid Res 1987;28:1110-7.

23. Esterbauer H, Gebicki J, Puhl H, Jürgens G. The role of lipid peroxidation and antioxidants in oxidative modification of LDL. Free Radical Biol Med 1992;13:341-90.

24. Rose GA, Blackburn H, Gillum RF, Prineas RJ. Cardiovascular Survey Methods. $2^{a}$ edição. Geneva: World Health Organization, anexo 1, 1982

25. Howard BV, Lee ET, Cowan LD, Fabsitz RR, Howard WJ, Oopik AJ, et al. Coronary heart disease prevalence and its relation to heart disease in American Indians: the Strong Heart Study. Am J Epidemiol 1995;142:254-68.

26. Décourt LV. Considerações sobre o intervalo QT. II. Limites superiores da normalidade. J Bras Med 1966;10:21.

27. Dekker JM, Crow RS, Hannan PJ, Schouten EG, Folson AR. ARIC Study. Heart rate-corrected QT interval prolongation predicts risk of coronary heart disease in black and white middle-aged men and women: the ARIC study. J Am Coll Cardiol 2004; $43: 565-71$

28. Snehalatha $C$, Ramachandran A, Sivasankari S, Satyavani K Viswanathan V, Misra J, et al. Is increased apoliprotein B-A major factor enhancing the risk of coronary artery disease in type 2 diabetes? J Assoc Physicians India 2002;50:1036-8.

29. Walldius G, Jungner I. Apolipoproteins B and apolipoprotein A-I: risk indicators of coronary heart disease and targets for lipid-modifying therapy. J Intern Med 2004;255:188-205.

30. Colhoum HM, Rubens MB, Underwood SR, Fuller JH. The effect of type 1 diabetes mellitus on the gender difference in coronary artery calcification. J Am Coll Cardiol 2000;36:2160-7. 
31. Kreisberg RA. Diabetic dyslipidemia. Am J Cardiol 1998:82:67U-73.

32. Hoeldtke RD, Bryner KD, McNeill DR, Hobbs GR, Riggs JE, Warehime SS, et al. Nitrosative stress, uric acid, and peripheral nerve function in early type 1 diabetes. Diabetes 2002;51:2817-25.

33. Kuyvenhoven JP, Meinders AE. Oxidative stress and diabetes mellitus. Pathogenesis of long-term complications. Eur J Intern Med 1999;10:9-19.

34. Steinberg D. Low density lipoprotein oxidation and its biological significance. J Biol Chem 1997;272:20963-6.

35. Tsai EC, Hirsch IB, Brunzell JD, Chait A. Reduced plasma peroxyl radical trapping capacity and increased susceptibility of LDL to oxidation in poorly controlled IDDM. Diabetes 1994; $43: 1010-4$.

36. Liguori A, Abete P, Hayden JM, Cacciatore F, Rengo F, Ambrosio G, et al. Effect of glycaemic control and age on lowdensity lipoprotein susceptibility to oxidation in diabetes mellitus type 1. Eur Heart J 2001;22:2075-84.

37. Jenkins AJ, Klein RL, Chassereau CN, Hermayer KL, LopesVirella MF. LDL from patients with well-controlled IDDM is not more susceptible to in vitro oxidation. Diabetes 1996:45:762-7.

38. Laakso M. Hyperglycemia and cardiovascular disease in type 2 diabetes. Diabetes 1999;44:937-42.

39. Stratton IM, Adler Al, Neil HA, Matthews DR, Manley SE, Cull $\mathrm{CA}$, et al. Association of glycaemia with macrovascular and microvascular complications of type 2 diabetes (UKPDS 35). BMJ 2000;321:405-12

40. Ceriello A. The emerging role of post-prandial hyperglycaemic spikes in the pathogenesis of diabetic complications. Diabet Med 1998; 15:188-93.
41. The DCCT Research Group. The effect of intensive treatment of diabetes on the development and progression of long-term complications in insulin-dependent diabetes mellitus. $\mathbf{N}$ Engl J Med 1993;329:977-86.

42. The Diabetes Control and Complications Trial/Epidemiology of Diabetes Interventions and Complications Research Group. Intensive diabetes therapy and carotid intima-media thickness in type 1 diabetes mellitus. N Engl J Med 2003;348:2294-303.

43. Yamasaki $Y$, Kawamori $R$, Matsushima $H$, Nishizawa $H$, Kodama M, Kubota M, et al. Asymptomatic hyperglycaemia is associated with increased intimal plus medial thickness of the carotid artery. Diabetologia 1995;38:585-91.

44. Hoeg JM. Lipoproteins and atherogenesis. Endocrinol Metab Clin 1998;27:569-84.

45. Nakaya N. Hypertryglyceridemia increase risk for CAD? Growing evidence suggests it plays a role. Postgrad Med 2000; $108: 77-84$.

Endereço para correspondência:

Simone Henriques de Castro

Rua Conde de Bonfim 422/301

20520-054 Rio de Janeiro, RJ

Fax: (21) 2234-2556

E-mail: sh.castro@uol.com.br 